\title{
SUSY at the ILC and Solving the LHC Inverse Problem
}

\author{
James S. Gainer ${ }^{1, *}$ \\ ${ }^{1}$ Stanford Linear Accelerator Center
}

Recently a large scale study of points in the MSSM parameter space which are problematic at the Large Hadron Collider (LHC) has been performed. This work was carried out in part to determine whether the proposed International Linear Collider (ILC) could be used to solve the LHC inverse problem. The results suggest that while the ILC will be a valuable tool, an energy upgrade may be crucial to its success, and that, in general, precision studies of the MSSM are more difficult at the ILC than has generally been believed.

\section{INTRODUCTION: THE LHC INVERSE PROBLEM}

As we all know, the LHC is scheduled to turn on this year. This means that we are entering (hopefully) an exciting era of discovery in fundamental physics. Obviously the biggest question is "Will the LHC discover new physics?"

Let's assume the answer to this question is "yes" (if the answer is "no" there will not be much for beyond-the-standard model phenomenologists to talk about). It would, of course, not be sufficient to know that there is new physics, we would want to learn as much about the new physics as possible.

In fact our this would not sate our curiosity. Any model of new physics contains some set of parameters and hence has some "parameter space". We would like to know the values of these model parameter, i.e. we would like to determine which point in the model's parameter space is realized in nature. It is our goal to determine how difficult this is to do.

Of course one cannot do this for every possible model of new physics; there are far too many. We will therefore specialize to the case of the Minimal Supersymmetric Standard Model, as SUSY is a very popular possibility for new physics, and the MSSM is the simplest supersymmetric extension of the standard model.

*Electronic address: jgainer@slac.stanford.edu 
Often when considering the MSSM one uses additional relations between parameters derived from a particular model of SUSY breaking. As we do not know which (if any) of these models is correct, we will consider the MSSM in essentially full generality.

However, the full MSSM has well over one hundred parameters. This gives us a parameter space with too many dimensions to sample randomly with any thoroughness. Fortunately, for many collider applications we only need to consider a smaller set of MSSM Lagrangian parameters:

- Weak scale gaugino masses

- Weak scale sfermion masses

- $\mu$ term

- $\tan \beta$

- Trilinear couplings $\left(A_{t, b, \tau}\right)$

This is still a large number of parameters (around 15-20, depending upon what assumptions one makes).

Following the work of Arkani-Hamed, Kane, Thaler, and Wang(AKTW)[1] in studying the "LHC Inverse Problem" (described below) we think of an experiment, or rather the interpretation of an experiment, as representing a mapping from an experimental signature (i.e. the data), which can be imagined as a point in the space of possible signatures, to a point in parameter space. This is a fancy way of saying that "in an experiment, one measures the parameters of the model which describes the physics."

There are different possibilities for the inverse mapping. (Here we are looking at to where the actual LHC signature, a point in possible LHC signature space, maps.) A given point in LHC signature space may correspond to a unique point in parameter space. This is the optimal scenario. It is also possible that a given point in the LHC signature space corresponds to a large region of parameter space. This means that the data does not significantly constrain the possible parameter space, or in simpler language that our measurements of model parameters have large error bars. Finally there is the interesting possibility that a given point in signature space maps to distinct, well-separated points in parameter space. We would describe this scenario as in "inverse problem". 
AKTW addressed the question of whether the MSSM has an inverse problem at the LHC. They

- generated 43,026 random points in an MSSM parameter space (we will term points in MSSM parameter space "models" from here on in a convenient abuse of terminology)

- generating $10 \mathrm{fb}^{-1}$ of Monte Carlo SUSY signal at LHC using PYTHIA[2]

- piped the signal through PGS[3] (fast detector simulation) and made histograms for hadron collider observables (signature)

- compared each pair of model signatures using a statistical test, to determine whether the signatures are distinguishable.

- NOTE: they did not include standard model backgrounds

To be more specific, to pick their models (points in parameter space) they chose the values of the parameters listed in Table 1 assuming uniform priors in the ranges listed.

Masses were kept below $1 \mathrm{TeV}$ in order to keep LHC cross sections reasonably high and from considerations of naturalness. The colored sparticles were kept heavier due to both theoretical preference and due to practical computing concerns (memory limitations).

They found that out of their 43,026 models there were 283 pairs of models whose signatures were indistinguishable (degenerate). These 283 pairs involved 383 models. Obviously some models were in more than one pair. This may seem like a small number. However for one to find this many degeneracies for the number of models generated suggests that each model would be degenerate with $\mathcal{O}(10-100)$ other points in parameter space.

\section{OUR PROJECT}

We considered the 383 models found to be in degeneracies at LHC (actually 242, since 141 models had a problem with the physical particle spectrum due to AKTW's use of PYTHIA as a spectrum calculator). We simulated these models at ILC (details follow) and compared ILC signatures of models in pairs at LHC. If for many of the pairs of models degenerate at LHC we find distinguishable signatures at ILC, then one could use the ILC to solve the LHC inverse problem. We find that this is only the case in about one third of pairs; the 
details of our study and conclusions are described below; more details can be found in the relevant papers [4][5].

\section{Details of Study}

To be more specific, we simulated SUSY signals at the $500 \mathrm{GeV}$ ILC for each of 242 models in LHC degeneracies using PYTHIA and CompHEP for the signal. We simulated $250 \mathrm{fb}^{-1}$ of integrated luminosity for each of $80 \%$ left and $80 \%$ right electron beam polarizations. In this we used a design specific beam spectrum. (Also considering positron polarization and the energy upgrade to $1 \mathrm{TeV}$ are works in progress.)

We also obtained 2 SM background samples which were generated by Tim Barklow[6] using WHIZARD/ O'Mega[7] and which used the same design specific beam spectrum as the signal These standard model backgrounds used full tree level matrix elements for all $2 \rightarrow 2,2 \rightarrow 4$, and $2 \rightarrow 6 \mathrm{SM}$ processes from each of $e e, e \gamma$ and $\gamma \gamma$ initial states. This includes over 1000 processes.

We then generated $\sim 25$ histograms of ILC observables for our signal and backgrounds using the SiD detector[8] simulation package org.lcsim[9]. To know what cuts to use in our (cut-based) analysis, we did an exhaustive literature search, but also improved on these cuts for our models by "fiddling around".

Once we had a number of histograms, representing different analyses, we used a log likelihood test to compare the signal for one model added to one background to a statistically independent Standard Model background. This allowed us to see whether that model's signature was "visible" at the $500 \mathrm{GeV}$ ILC. Note: We did this for each histogram, so we know whether each produced sparticle is visible for each model, and in what analyses it is visible.

We also used a $\chi^{2}$ test to compare a model's signature plus a standard model background to a different model (with a degenerate LHC signature) plus a statistically independent standard model background, to see if (for each histogram) the models yield distinguishable signatures. 


\section{A. Kinematic Accessibility of Sparticles}

Of our 242 models, 181 have sparticles accessible at $500 \mathrm{GeV}$. 85 have charged sparticles accessible at $500 \mathrm{GeV}$. These models have a total of 140 accessible charged sparticles. At 1 $\mathrm{TeV}$, all but 1 of the 242 models have accessible sparticles; many more charged sparticles are also accessible at this energy. Further details on the kinematic accessibility of various sparticles is given in Table 2.

\section{B. Backgrounds and Cuts}

Since we used full SM backgrounds, including all $e^{+} e^{-}, e \gamma$ and $\gamma \gamma$ initial states, and considered the fully SUSY signal for each analysis, we had large backgrounds. It was therefore essential to cut backgrounds substantially while retaining as much signal as possible. This is shown for our selectron search in Figure 1.

\section{RESULTS AND CONCLUSIONS}

Figure 2 shows the histograms obtained from one of our selectron searches for several of our models as well as for the benchmark point SPS1a'[11]. Many of the salient results of our paper can be seen here. The two-level shelf shape of the signal from the bench mark point SPS1a' is due to the fact that both left and right selectrons are light in that model.

Note that most of our models have much lower cross sections than SPS1a'. It seems that the use of optimistic benchmark points makes the detailed determination of SUSY parameters at ILC look easier than it really will be.

We find that we can distinguish, at the $5(3) \sigma$ confidence level, 57(63)/72 pairs of models where at least one of the models has a charged spartner kinematically accessible, but we fail completely when both models being compared have only neutral spartners accessible. Thus, out of all of the AKTW model pairs, our results show that 57(63)/162 can be distinguished at the ILC $5(3) \sigma$.

Our results as far as model visibility and the distinguishing of LHC pairs are shown in Table 3. We see the ILC will be useful (it distinguishes models with degenerate LHC signatures) but not in all cases. This is in contrast to "standard lore" about the ILC; the difference is largely due to our consideration of especially realistic backgrounds and the 
kinematic limitations of a $500 \mathrm{GeV}$ ILC. This suggests that upgrading the ILC energy as soon as possible may be essential to fully capitalize on discoveries made at the LHC.

\section{Acknowledgments}

The work desribed was supported in part by the United States Department of Energy, Contract DE-AC02-76SF00515. The author wishes to thank the organizing committee of the 11th International Moscow School Of Physics and 36th ITEP Winter School of Physics for their generous hospitality. 
[1] N. Arkani-Hamed, G. L. Kane, J. Thaler and L. T. Wang, JHEP 0608, 070 (2006) [arXiv:hep$\mathrm{ph} / 0512190]$.

[2] T. Sjostrand, S. Mrenna and P. Skands, JHEP 0605, 026 (2006) [arXiv:hep-ph/0603175].

[3] PGS: Simple simulation package for generic collider detectors, by J. Conway; http://www.physics.ucdavis.edu/ conway/research/software/pgs/pgs.html.

[4] C.F. Berger, J.S. Gainer, J.L. Hewett, B. Lillie and T.G. Rizzo, arXiv:0711.1374 [hep-ph].

[5] C.F. Berger, J.S. Gainer, J.L. Hewett, B. Lillie and T.G. Rizzo, arXiv:0712.2965 [hep-ph].

[6] The SM backgrounds we employ here have been generated by T. Barklow.

[7] W. Kilian, T. Ohl and J. Reuter, arXiv:0708.4233 [hep-ph], http://www-ttp.physik. uni-karlsruhe.de/ whizard/.

[8] Silicon Detector Concept, http://www-sid.slac. stanford.edu/

[9] http://www.lcsim.org/

[10] E. Goodman, The Search for the Supersymmetric Selectron, April 15, 1998, COLO-HEP-398, unpublished.

[11] J. A. Aguilar-Saavedra et al., Eur. Phys. J. C 46, 43 (2006) [arXiv:hep-ph/0511344]; B. C. Allanach et al., in Proc. of the APS/DPF/DPB Summer Study on the Future of Particle Physics (Snowmass 2001) ed. N. Graf [arXiv:hep-ph/0202233]. 

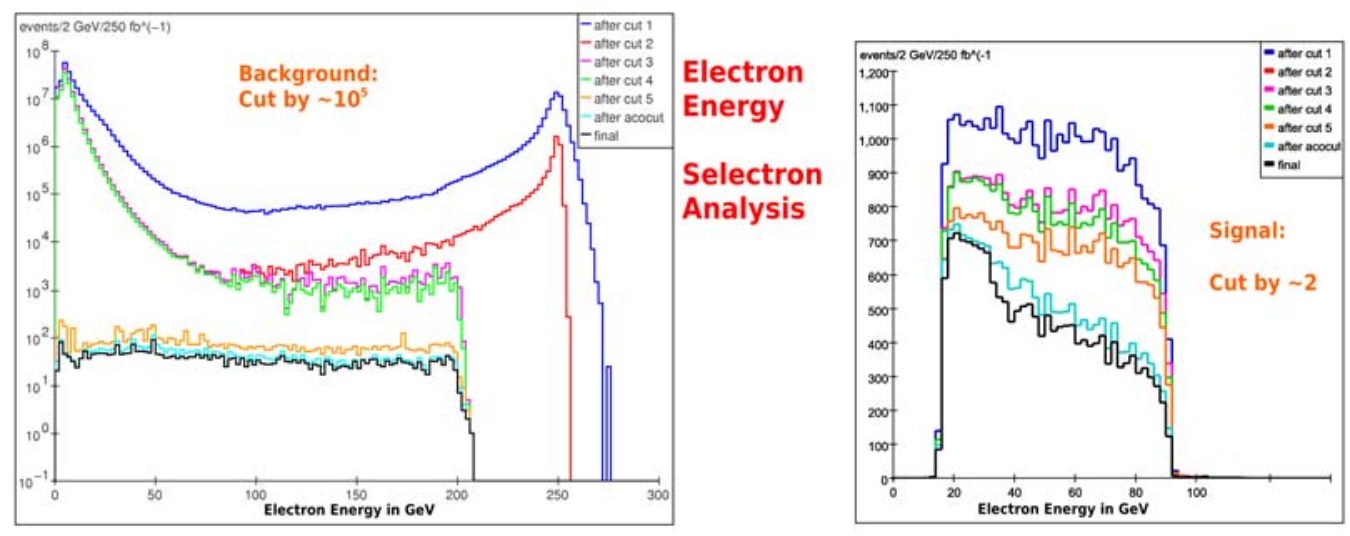

FIG. 1: We had to cut selectron backgrounds by (a lot), while signal by as little as possible. Our cuts for this analysis were largely derived from those of Goodman[10]. 


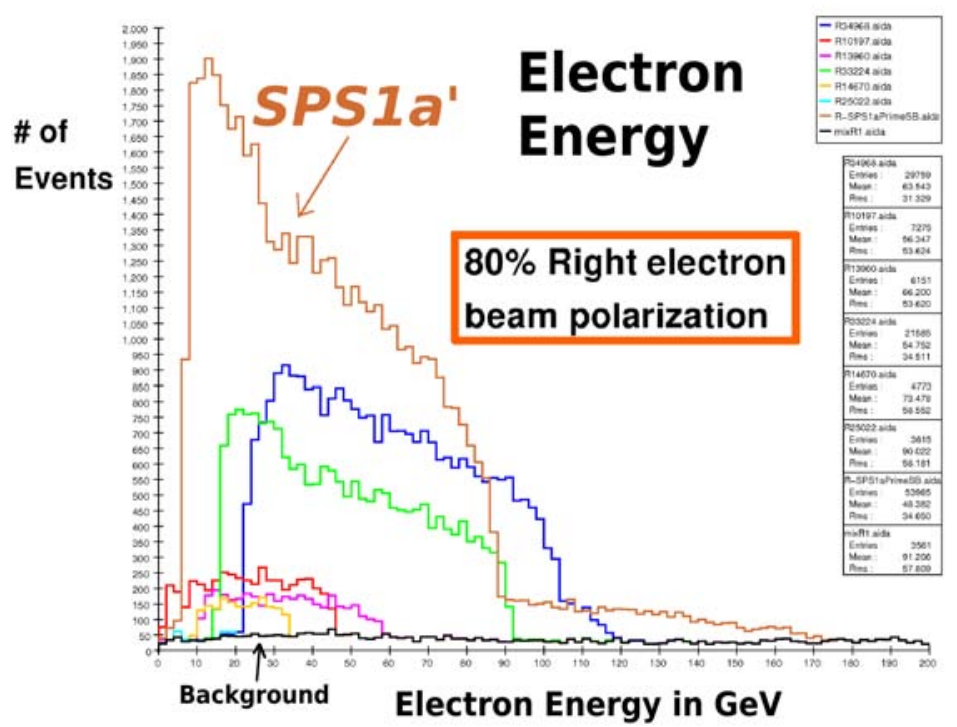

FIG. 2: This figure shows the energy of electrons (and positrons) from various of our models (the intermediate curves), as well as the benchmark point SPS1a'[11]. Number of events is given on the $\mathrm{y}$-axis, while the energy of the electron or positron is given on the $\mathrm{x}$-axis. 
TABLE I: The MSSM parameter ranges used for selecting random points in MSSM parameter space ("models") in [1].

\begin{tabular}{|c|c|c|}
\hline Parameter & Min. & Max. \\
\hline$M_{1}, M_{2}, \mu$ & $100 \mathrm{GeV}$ & $1 \mathrm{TeV}$ \\
$m_{l_{1,2}}, m_{e_{1,2}}, m_{l_{3}}, m_{e_{3}}$ & $100 \mathrm{GeV}$ & $1 \mathrm{TeV}$ \\
$M_{3}$ & $600 \mathrm{GeV}$ & $1 \mathrm{TeV}$ \\
$m_{q_{1,2}}, m_{u_{1,2}}, m_{d_{1,2}}$ & $600 \mathrm{GeV}$ & $1 \mathrm{TeV}$ \\
$m_{q_{3}}, m_{u_{3}}, m_{d_{3}}$ & $600 \mathrm{GeV}$ & $1 \mathrm{TeV}$ \\
$\tan \beta$ & 2 & 50 \\
$M_{A}$ & $800 \mathrm{GeV}$ & $800 \mathrm{GeV}$ \\
$A_{\tau}$ & 0 & 0 \\
$A_{b}, A_{t}$ & $850 \mathrm{GeV}$ & $850 \mathrm{GeV}$ \\
\hline
\end{tabular}


TABLE II: The table shows the number of models with a given final state kinematically accessible at the given energies.

\begin{tabular}{|c|c|c|}
\hline Final State & $500 \mathrm{GeV}$ & $1 \mathrm{TeV}$ \\
\hline$\tilde{e}_{L}^{+} \tilde{e}_{L}^{-}$ & 9 & 82 \\
\hline$\tilde{e}_{R}^{+} \tilde{e}_{R}^{-}$ & 15 & 86 \\
\hline$\tilde{e}_{L}^{ \pm} \tilde{e}_{R}^{\mp}$ & 2 & 61 \\
\hline$\tilde{\mu}_{L}^{+} \tilde{\mu}_{L}^{-}$ & 9 & 82 \\
\hline$\tilde{\mu}_{R}^{+} \tilde{\mu}_{R}^{-}$ & 15 & 86 \\
\hline Any selectron or smuon & 22 & 137 \\
\hline$\tilde{\tau}_{1}^{+} \tilde{\tau}_{1}^{-}$ & 28 & 145 \\
\hline$\tilde{\tau}_{2}^{+} \tilde{\tau}_{2}^{-}$ & 1 & 23 \\
\hline$\tilde{\tau}_{1}^{ \pm} \tilde{\tau}_{2}^{\mp}$ & 4 & 61 \\
\hline$\tilde{\nu}_{e \mu} \tilde{\nu}_{e \mu}^{*}$ & 11 & 83 \\
\hline$\tilde{\nu}_{\tau} \tilde{\nu}_{\tau}^{*}$ & 18 & 83 \\
\hline$\tilde{\chi}_{1}^{+} \tilde{\chi}_{1}^{-}$ & 53 & 92 \\
\hline Any charged sparticle & 85 & 224 \\
\hline$\tilde{\chi}_{1}^{ \pm} \tilde{\chi}_{2}^{\mp}$ & 7 & 33 \\
\hline$\tilde{\chi}_{1}^{0} \tilde{\chi}_{1}^{0}$ & 180 & 236 \\
\hline$\tilde{\chi}_{1}^{0} \tilde{\chi}_{1}^{0}$ only & 91 & 0 \\
\hline$\tilde{\chi}_{1}^{0}+\tilde{\nu}(\bar{\nu})$ only & 5 & 0 \\
\hline$\tilde{\chi}_{1}^{0} \tilde{\chi}_{2}^{0}$ & 46 & 178 \\
\hline No sparticles & 61 & 3 \\
\hline
\end{tabular}


TABLE III: Number of models, at $\sqrt{s}=500 \mathrm{GeV}$, which have a given final state particle visible above the SM background with a significance $\mathcal{S}>5$ divided by the number of models with the same particle kinematically accessible.

\begin{tabular}{|c|c|}
\hline \hline Particle & Number Visible \\
\hline$\tilde{e}_{L}$ & $8 / 9$ \\
$\tilde{e}_{R}$ & $12 / 15$ \\
$\tilde{\mu}_{L}$ & $9 / 9$ \\
$\tilde{\mu}_{R}$ & $12 / 15$ \\
$\tilde{\tau}_{1}$ & $21 / 28$ \\
$\tilde{\nu}_{e, \mu}$ & $0 / 11$ \\
$\tilde{\nu}_{\tau}$ & $0 / 18$ \\
$\tilde{\chi}_{1}^{ \pm}$ & $49 / 53$ \\
$\tilde{\chi}_{1}^{0}$ & $17 / 180$ \\
$\tilde{\chi}_{2}^{0}$ & $5 / 46$ \\
\hline \hline
\end{tabular}

